\title{
Intestinal gas
}

By M. D. LevitT, ACOS for Research, Veterans Administration Medical Center,

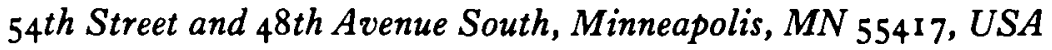

When the patient complains of 'too much gas', he or she may actually be referring to one of three complaints: excessive eructation, bloating and abdominal pain (which he attributes to too much gas in the gut), or excessive passage of flatus. The physician must differentiate between these three conditions because they have different etiologies and different therapies.

\section{Chronic eructation}

The isolated belch actually removes gas from the stomach as evidenced by a decrease in the size of the gastric air-bulb. In contrast, chronic eructation does not remove gas from the stomach. Rather the subject precedes each chronic belch with a manoeuvre which relaxes the upper oesophageal sphincter at the same time as a negative pressure is produced in the chest. By this means, air is 'sucked' into the oesophagus. Most of this air is immediately regurgitated but some may actually pass on down into the stomach. The chronically-belching subject may therefore have a large gastric air-bubble but there should be no confusion: the gastric air-bubble is large because the patient is belching, the patient is not belching because of large quantities of gastric gas. Further evidence that this air-bubble is swallowed is provided by analysis of this gas which shows it to have a composition comparable to air with only minor quantities of gases such as carbon dioxide, hydrogen or methane which are produced in the gut. Given this origin of chronic belching, it is totally irrational to treat these patients with drugs aimed at the gastrointestinal tract such as anticholinergics, antacids or simethicone. Rather, the only therapy that has a hope of permanent success is the instruction of the patient on the origin of his problem with the admonition to resist the urge to belch.

\section{Bloating and abdominal pain}

Although millions of patients attribute their symptom to the presence of excessive gas in the gut, there is little evidence to support the idea that too much gas is the usual cause of their complaint. We measured the volume of gas in the intestine of controls and bloating patients by having them swallow a tube which was allowed to pass to the ligament of Treitz and argon gas was then rapidly perfused though this tube. We assumed that as the Ar passed through the bowel, it would wash out all the gases native to the gut $\left(\mathrm{H}_{2}\right.$, oxygen, $\mathrm{CO}_{2}$, nitrogen and 
$\mathrm{CH}_{4}$ ). By collecting all gas washed out at the rectum and analysing this gas for the five gases native to the gut, we determined the volume and composition of the gas in the intestinal pool.

This technique showed that healthy controls and bloating patients had similar volumes of bowel gas (about $200 \mathrm{ml}$ ) and there was no difference in the composition of this gas when controls and bloating subjects were compared. Thus, we found no evidence that abnormal volumes or composition of intestinal gas are the basis of the complaint of bloating and distension.

We did find, however, that bloating patients had far more severe pain during the Ar infusion than did controls. In addition, the bloating patients refluxed more $\mathrm{Ar}$ back into the stomach. Thus, the primary abnormality in such patients appears to be a motility disorder which causes these patients to experience pain when their guts are distended by volumes of gas which were tolerated by normal subjects.

Presumably, the therapy of such subjects should be directed towards correcting the apparent disorder of motility. Unfortunately anticholinergics are of limited value in this regard. There is some evidence that drugs which stimulate motility, such as metoclopramide, may be useful.

\section{Passage of excessive flatus}

Analysis of flatus of most subjects complaining of excessive rectal gas shows that $\mathrm{N}_{2}$ and $\mathrm{O}_{2}$, the gases that can be swallowed, makes up less that $50 \%$ of the gas. Rather, gases produced in the gut $\mathrm{H}_{2}, \mathrm{CO}_{2}$ and sometimes $\mathrm{CH}_{4}$, comprise the majority of rectal gas. Thus gas production, not air-swallowing, is the source of the problem.

The production of $\mathrm{H}_{2}$ and $\mathrm{CO}_{2}$ in the colon appears to be largely dependent on the fermentation of carbohydrate by the colonic bacteria. In order for the colonic bacteria to gain access to such carbohydrate, the small bowel must incompletely absorb some ingested carbohydrate. There is a variety of carbohydrates which may make their way to the colon of healthy subjects including lactose in lactase-deficient subjects, and foods which contain non-absorbable carbohydrates such as legumes and wheat.

The only presently feasible method to reduce flatus production at the present time appears to be the use of a diet that reduces the quantity of carbohydrate reaching the colon: namely a lactose-free, low-wheat diet. 